\title{
Editorial: About This Issue
}

T The first issue of JCOMSS presents a selection of papers originally presented at the conference on Software, Telecommunications and Computer Networks SoftCOM 2004. The conference was held in October 2004 aboard the cruising ship Marko Polo on the route Split-Venice-Dubrovnik under the technical co-sponsorship of the IEEE Communications Society (ComSoc). In the CfP there was an announcement for publication of the best ranked papers. Twenty-five best papers ranked by international reviewers have been proposed for publication. Twenty-two papers have been submitted and eight of them have passed the standard reviewing process. Papers published in the first issue of JCOMMS cover a number of interesting topics in the areas of communications software, communication networks and protocols, wireless systems, and multimedia signal processing.

In the paper addressing the area of communications software, "The Two-Step P2P Simulation Approach A Framework for Message- and Packet-Level Simulation" by Birck et al., a framework for analysis of the effects \& requirements of $\mathrm{P} 2 \mathrm{P}$ applications on application and on network layer has been introduced. P2P applications are complex and deployed on a large scale, pure packet level simulations do not scale well enough to analyze P2P applications in a large network with thousands of peers. It is also difficult to assess the effect of application level behavior on the communication system. The authors propose an approach starting with a more abstract and therefore scalable application level simulation.

There are three papers addressing the area of communication networks and protocols. In "A New Perspective for a Global QoS-based Internet" Levis et al. demonstrate that it is possible to provision QoS-enabled services that maintain the ability to easily connect any pair of users worldwide. Several Quality of Service (QoS) architectures and mechanisms have been proposed by standardisation bodies. Most research activities have focused to date on supporting QoS only within a single administrative domain. For this purpose, the authors introduce the notion of Meta-QoS-Class and demonstrate its relevance for building QoS-enabled services across multiple domains. This concept opens up a new perspective for a global QoSbased Internet.

In "A Model-based Scalable Reliable Multicast Transport Protocol for Satellite Networks" Chumchu et al. design a new scalable reliable multicast transport protocol for satellite networks (RMT). The proposed protocol is divided in 2 parts: error control part and congestion control part. In error control part, we intend to solve feed back implosion and improve scalability by using a new hybrid of ARQ (Auto Repeat Request) and adaptive forward error correction (AFEC). wireless link utilization used by the proposed protocol is virtually independent of number of multicast receivers. In congestion control part, the proposed protocol employs a new window-based congestion control scheme, which is optimized for satellite networks.

In "A Framework for Performance Analysis of Ad Hoc Networks and Its Application to a Slotted-ALOHA Scenario" Granelli proposes a framework to analyze the problems of connectivity and performance in ad-hoc networks through an analytical approach. To this aim, available results related to the application of the percolation theory to the study of connectivity in ad-hoc network are exploited jointly with communication theory models. The framework is then applied to a slotted ALOHA ad-hoc network. Theoretical and numerical results validate the approach.

The next two papers address the area of wireless systems. In "Design and Implementation of a Low Complexity Multiuser Detector for Hybrid CDMA Systems“ Rampa compares multiuser detectors (MUD) for Hybrid CDMA mobile radio systems based on the CWTS standard. The author introduced in a previous paper a novel MUD algorithm based on the Bareiss algorithm; here it is compared with other well known detectors (e.g. block-Levison, block-Fourier) in terms of performance, complexity and hardware feasibility. Low level simulation results for both block-Bareiss and BlockFourier are presented and commented.

In "Efficient Algorithmic and Architectural Optimization of QR-based Detector for V-BLAST" Sobhanmanesh and Nooshabadi investigate the use of multiple antennas at both transmitting and receiving sides of a rich scattering communication channel to improve the spectral efficiency and capacity of digital transmission systems compared with single antenna communication systems. Algorithmic complexity in the realization of the receiver is a major problem for its implementation in hardware. The authors analyze a near optimal algorithm for V-BLAST detection in MIMO wireless communication systems based on the $\mathrm{QR}$ factorization technique, offering remarkable reduction in the hardware complexity. In particular some hardware implementation aspects of the selected algorithm have been analyzed through MATLAB simulations. This technique can be used in an efficient fixed point VLSI implementation of the algorithm. The VLSI architecture that implements the algorithm has been presented.

The last two papers address the area of multimedia signal processing. In "Video Smoothing of Streams Aggregate with Bandwidth Constraints" Camarda et al. introduce a novel smoothing algorithm. Compressed variable bit rate (VBR) video transmission is assuming a growing importance in the telecommunication world. High data rate variability of compressed video over multiple time scales makes an efficient bandwidth resource utilization difficult to obtain. Smoothing techniques is one of the approaches developed to face this problem. The proposed smoothing algorithm represents a significant improvements over existing methods. It performs 
data scheduling both for a single stream and stream aggregations, by taking into account available bandwidth constraints.

In "Power Constrained Space-Time Processing for Suppression of Electromagnetic Fields" Hult and Mohammed investigate the possibility of applying the adaptive active control methods with the aim of lowering the electromagnetic field power density at a specific volume in space using the superposition principle and space-time processing employing the full MIMO (Multiple Input Multiple Output) antenna system setup. The application that the authors evaluated is a model of a mobile phone equipped with one ordinary transmitting antenna and two actuator antennas which purpose is to cancel out the electromagnetic field at the human head while maintaining a predefined level of the overall output power of the system. Simulation results show the promise of using the adaptive control algorithms and MIMO system to attenuate the electromagnetic field power density.
In conclusion, Editors sincerely hope that this first issue of JCOMSS does provide an up-to-date and valuable research information on an interesting selection of topics relevant to area of communications software and systems. We also hope that it will inspire the authors to submit their papers for publishing in future JCOMSS issues. We would like to thank all the authors who submitted their papers, and thank all the reviewers for their efforts and valuable contributions. We are grateful to Steve. B. Weinstein for his valuable advices and suggestions.
Editors-in-Chief:

DINKO BEGUSIC

begusic@fesb.hr

ALGIRDAS PAKSTAS

a.pakstas@ieee.org

MLADEN KOS

mladen.kos@fer.hr 